\title{
Towards a Semantically-Enriched Framework for Human Resource Management
}

\author{
D. Arena ${ }^{1}$, K. Ziazios ${ }^{2}$, IN. Metaxa ${ }^{2}$, S. Parcharidis ${ }^{3}$, S. Zikos ${ }^{4}$, A. Tsolakis ${ }^{4}$, S. \\ Krinidis $^{4}$, D. Ioannidis ${ }^{4}$, D. Tzovaras ${ }^{4}$, D. Kiritsis ${ }^{1}$ \\ ${ }^{1}$ École Politechnique Fédérale de Lausanne (EPFL), \\ SCI-STI-DK ME, Station 9, CH-1015 Lausanne, Switzerland \\ ${ }^{2}$ Atlantis Engineering S.A., Greece \\ ${ }^{3}$ Systems Sunlight S.A., Greece \\ ${ }^{4}$ Centre for Research and Technology Hellas- Information Technologies Institute (CERTH/ITI), \\ Thessaloniki, Greece \\ damiano.arena@epfl.ch, \{ziazios, metaxa\}@abe.gr, \\ s.parcharidisesunlight.gr, \{szikos, tsolakis, krinidis, \\ djoannid, dimitrios.tzovaras\}eiti.gr, dimitris.kiritsiseepfl.ch
}

\begin{abstract}
Human resources are one of the most important assets of an organization. The setup of a proper Human Resource Management system undoubtedly represents one of the pillars upon which any organization should be built. Many effective standards and solutions have been proposed in the past decades. However, the ever changing environment and the emerging technologies, such as ontologies and linked data, lead to adapt them and consider new approaches. The solution proposed in this document aims to combine existing standards for manufacturing information and ontology modelling. As a result, the development of an ontology model enhancing the HR information flow with semantics, on the one hand, enables the use of common data formats and exchange protocols promoted by the world Wide Web Consortium (W3C) and exploitable on the Sematic Web. On the other hand, it lays the foundations for an automated decision making process based on inference rules and smart data management. A study has been performed in a real-life industry revealing highly notable results.
\end{abstract}

Keywords: Human resources $\bullet$ B2MML • Ontology • Semantic Interoperability

\section{Introduction}

Today more than ever, companies are seriously considering to adopt strong and effective Human Resource Management (HRM) solutions in terms of HR analysis capabilities and smart information management. Factories are moving towards the future, but the HRM solutions need more effort to keep up. In a changing manufacturing and operating environment with human-machine interactions, using the possibilities offered by the Internet of Things and real-time or near real-time data, that result to big data, 
things are getting more and more complicated. More advanced HRM products, compared to more conventional ones, such as Customer Relationship Management (CRM) and Enterprise Resource Planning (ERP) systems, appear nowadays in the market. The big players, like SAP or IFS, offer solutions, along with medium range ones, such as JAMS, Automic, StreetSmart, SmartFactory and more. This is a living proof of a concise need of the market. However, it should be noted that most of the products are oriented towards a specific niche market or they are focused on interconnections and integrations with specific Manufacturing Execution Systems (MES). Hence, there is a need for a data exchange model based on existing standards and emerging technologies that can translate business procedures and manufacturing operations to information that can be exploited for dynamic HRM solutions. In this work, elements from the Business To Manufacturing Markup Language schema (B2MML) have been translated into elements of an owl ontology to provide a framework aiming to enrich the semantics of the B2MML-based data flow, enabling knowledge sharing and semantic interoperability through the Semantic Web [1].

\section{B2MML}

HRM can be considered as one of the most complex tasks, especially in industrial environments, when dealing with resource management, due to the highly dynamic attributes that present themselves in real-time operation, such as machine failures, the advent of urgent jobs, etc. To facilitate the information exchange at environments that host a vast heterogeneous network of distributed information sources that constantly produce data in various formats and intervals, a Common Information Data Exchange Model (CIDEM) has been introduced [2], with one of the core components being the B2MML schema.

The B2MML, as published by the Manufacturing Enterprise Solutions Association (MESA [3]), is an open XML representation of the Enterprise-Control System Integration standard(s) (ANSI/ISA-95 [4]), and it is used to specify and constrain the content of the information flow between Enterprise and Control systems [5], and specifically describes XML schemas for four information categories: a) Capability \& Capacity Definition, b) Product Definition, c) Production Schedule, and d) Production Performance, as well as four resource categories: i) Personnel, ii) Equipment, iii) Material and Energy, and iv) Process Segments.

As depicted in Fig.1, the use of B2MML schemas can offer an open, interoperable, vendor independent and straight forward data exchange at production lines, optimally linking ERP and supply chain management systems with manufacturing systems such as Industrial Control Systems and MES. As such, and within the scope of the presented work, the B2MML schema was used as the foundation on which the information exchange was based upon; delivering a B2MML ontology that enables the formation of the semantically-enriched framework that enhances human resource management at production lines. 


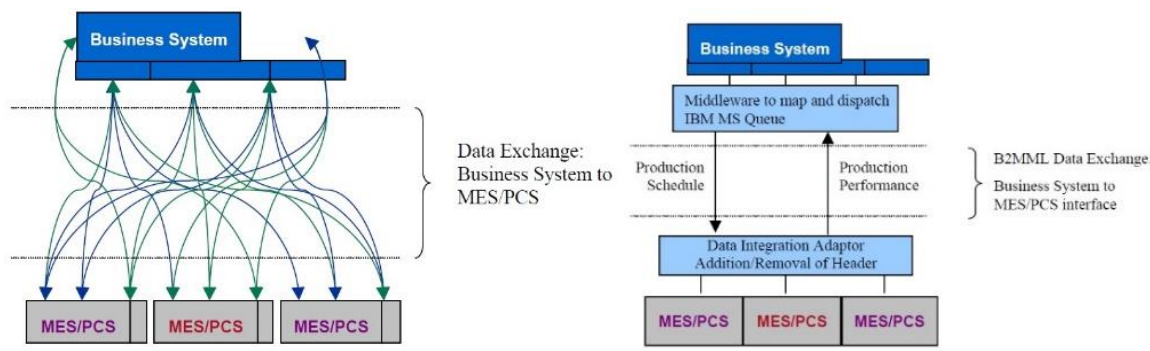

Fig. 1. Shop floor information exchange architecture before and after the deployment of the ISA-95 standard and B2MML schemas

\section{$3 \quad$ Semantic Modelling}

Industrial assets - in particular human resources - management is gaining from the use of semantic technologies and, in particular, knowledge base development [6] [7] [8]. Semantics structures, such as ontology models, provide a formal and ubiquitous information artefact aiming to make all the elements of a domain and their links explicit. Indeed, ontologies play an important role for many knowledge-intensive applications since they provide formal representations of domain knowledge, leveraging many of the properties discussed in [9].

According to the proposed approach, elements (e.g. classes and properties) from the B2MML xml schema have been, therefore, translated into elements of the B2MML owl ontology. Semantics is initially extracted from the B2MML xml schema and used as a basic taxonomy describing the former's classes. Relations among such classes have been defined in a way that each triplet appears as follows:

\section{([DomainClassName] ; has[RangeClassName] ; [RangeName])}

Three more (enumerated) classes have been, then, further characterized to meet the requirements specification set by the specific domain of analysis. In particular, PriorityLevel \{Non_Critical, Basic, Critical\}, ActorGroup \{MaintenanceSupervisor, Process, Operator, FloorManager, AutomationTechnician, ProcessSupervisor, ProcessTechnician, MaintenaceManager, ElectricalTechnician\}, Experiencelevel \{Experienced, Novice, Trainee\}. Eventually, a thorough analysis of the domain, in particular of the actor group mentioned above, allowed the extraction of 26 standard skills that can describe the requirements of each specific worker (actor) group. The results of this analysis were embedded in the ontology model. In fact, a further extension of basic semantic structure and subsequent alignment has been carried out by adding the enumerated class Skill \{Skill1, Skill2, ..., Skill26\} (Fig.2). 


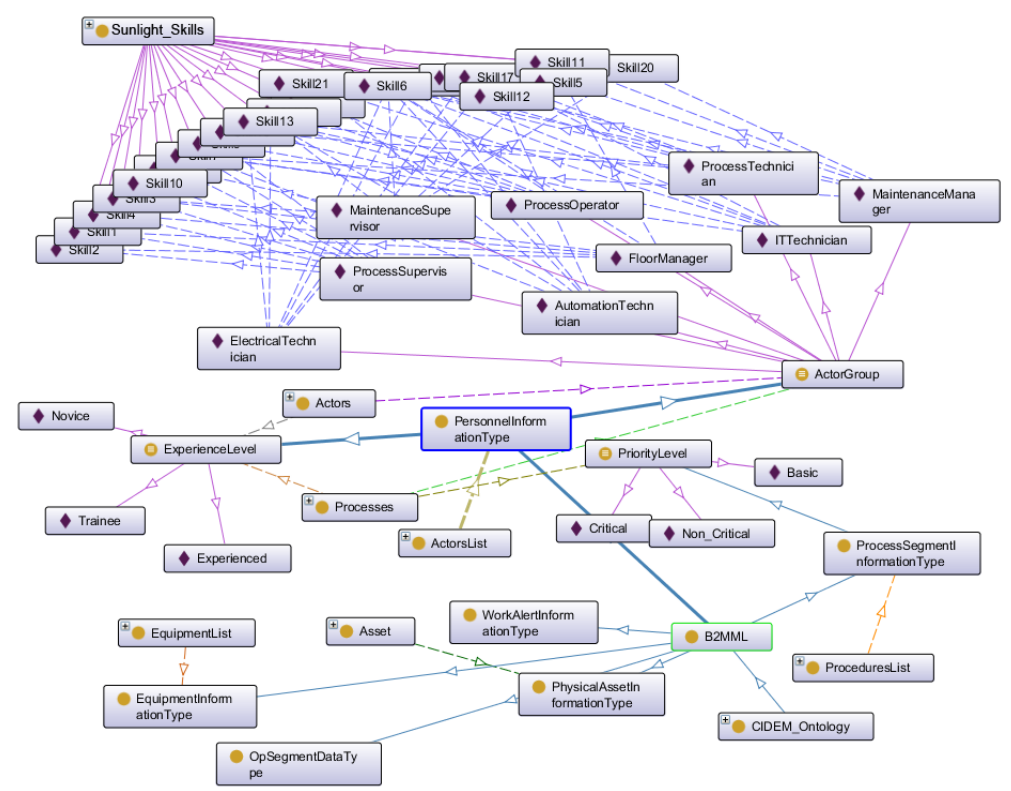

Fig. 2. Extension of the basic semantics with "skill" concept \& instances

The semantically-enriched skills-based description of the worker groups enables the creation of inference rules to correlate worker skills to groups for each worker's profile. Moreover, it provides an enriched structure that may continuously grow and discover new knowledge.

$$
\begin{gathered}
\text { hasSkill some Skill and hasSkill only (Skill[1], ..., Skill[n]) } \rightarrow \\
\text { hasActorGroup(Actor, [ActorGroup]) }
\end{gathered}
$$

\section{Semantics-based HR information management}

In order to be able to utilise information stored in CIDEM, B2MML based static and dynamic data has to be transformed into RDF/XML (Resource Description Framework) [10] triplets by employing EXtensible Stylesheet Language Transformation (XSLT) as a means to map XML and OWL elements and execute the actual data transformation (Fig. 3), according to the W3C Recommendation for defining XML data transformation and presentation. The RDF standard was also developed by the $\mathrm{W} 3 \mathrm{C}$ and helps to create metadata for describing web resources, enabling their sharing through the Semantic Web [11]. 


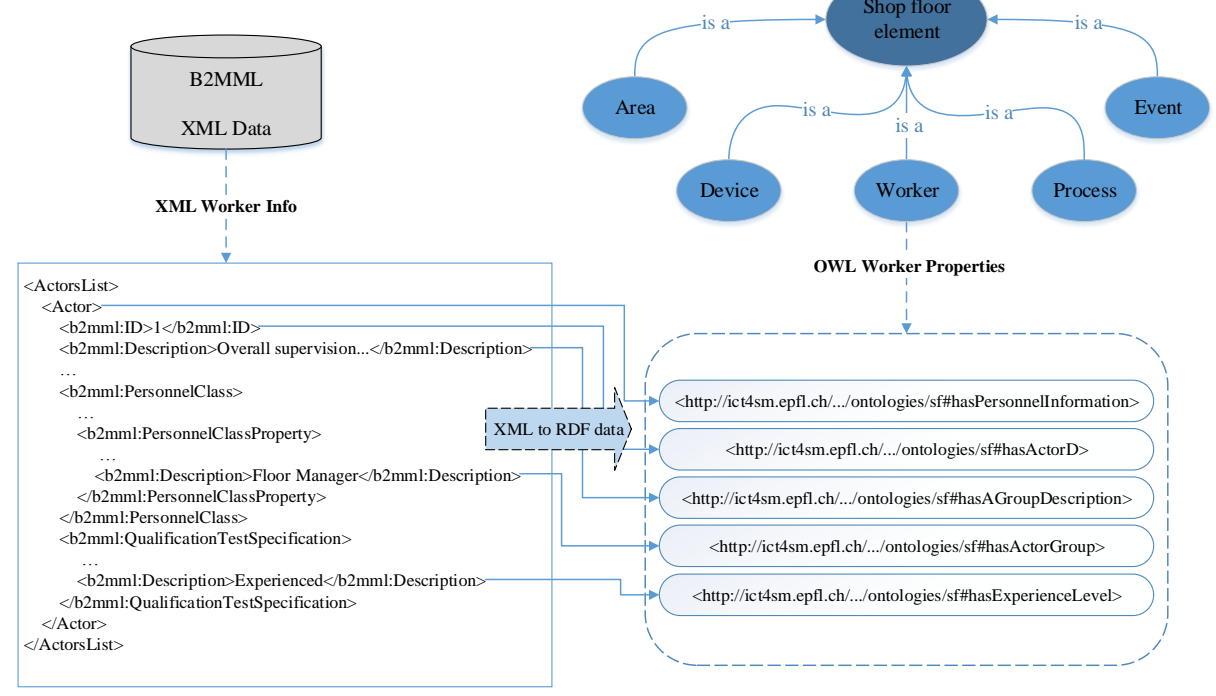

Fig. 3. From $X M L$ to RDF

SPARQL (SPARQL Protocol and RDF Query Language) [12] can be therefore exploited to express queries across the obtained RDF data sources, enabling a semanticsenhanced HR information browsing and knowledge management.

In particular, the efficient management of human resources and the optimization of the allocation of workers according to the needs of an organization are extremely important in fulfilling both the organization's objectives and the workers' feeling of satisfaction. The semantically-enriched information produced by the proposed framework can be exploited in different application scenarios. For instance, the suitability level of a worker to perform a specific task, which is described according to the model applied, can be evaluated using the semantic information. Assigning a task to the suitable worker according to his/her skills can result in the total task execution makespan. Furthermore, the availability of the automatically generated semantic information about HR and tasks paves the way towards automated or suggestions for actions regarding the assignments of tasks. This benefit can be important in highly dynamic environments, where for example, new maintenance operations can occur due to unexpected critical machine failures, and immediate response to handle the malfunction by the appropriate personnel is required. Another practical application of the produced semantically-enriched information is its presentation to the HR supervisor in a user-friendly human-readable format, in order to provide useful insights about the performance of workers, their skills, and their progress. 
Regarding the utilisation of semantic information to perform automated actions, a decision-making engine could perform the following indicative operations:

a. Propose the most suitable worker to perform a new arriving task;

b. Use the semantic information as one of various parameters in order to make realtime intelligent decisions and schedule new tasks based on multiple criteria; and

c. Balance the workload among workers efficiently by taking into account their skills and the characteristics of the scheduled tasks.

\section{$5 \quad$ Use Case}

This approach has been applied to a real industrial shop floor environment at Systems Sunlight S.A. in the framework of the H2020 funded SatisFactory project. Sunlight is a global player in the field of integrated energy solutions, specializing in the development, production and marketing of batteries and energy storage systems for industrial, advanced technology and consumer applications. The challenge was to integrate data from multiple production cells and/or lines, to account for different types of actors and operators (also in terms of skills and organizational level), to consider different levels of expertise of employees, to combine information from the localization system of the employees in the shop floor and to integrate with the solution package suitable for the execution of a certain task. Here, the ontology framework is used to infer the time for each worker to perform a desired job. Based on the evaluation step, for all next steps the outcome is evaluated by the Decision Support System (DSS) through an HR optimisation process that leads to an improved workload balancing as can be seen in Fig. 4.

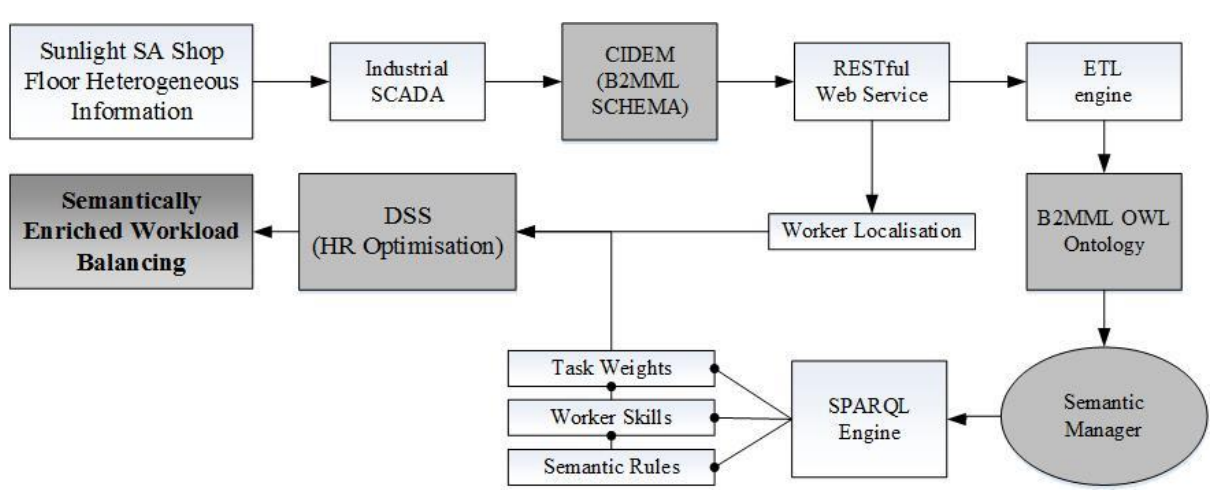

Fig. 4. Data exchange flow at Sunlight SA Shop Floor

According to the given problem, the duration for each task may vary, depending on the type of tasks and the skills of the worker that is assigned to carry out that task. Therefore, semantic rules are used to infer the expected execution time for a certain task, taking into account both workers' skills and task's requirements. Eventually, the ontology framework produces a time estimation as shown in Fig. 5. 


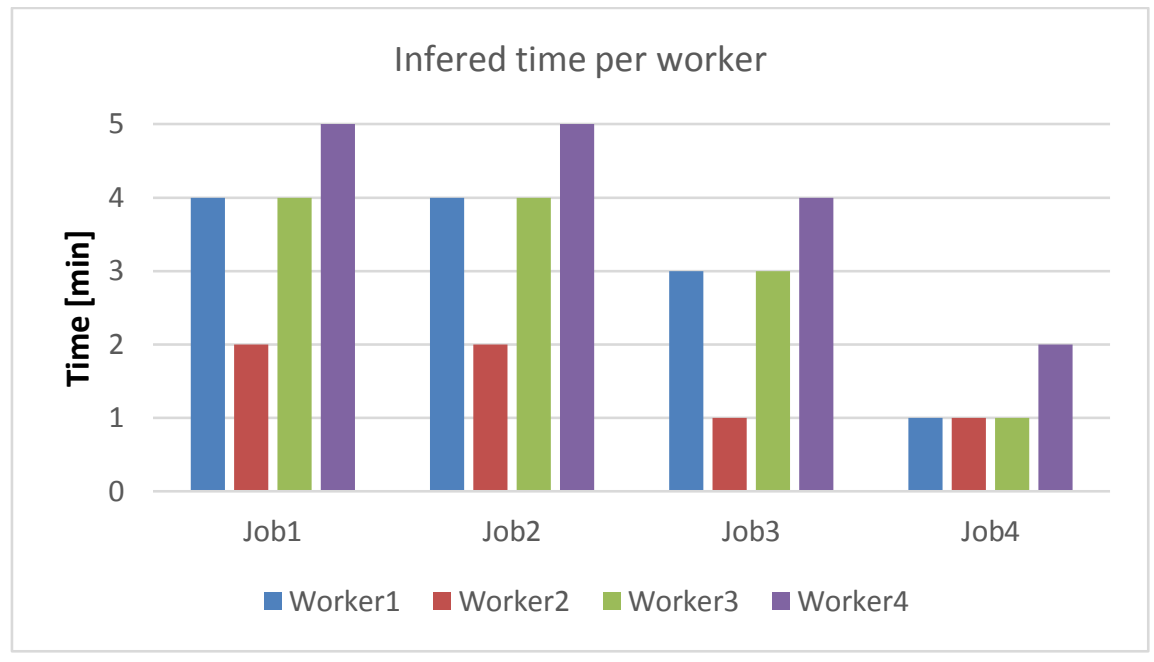

Fig. 5. Time estimation per worker

\section{Conclusions}

Elements of the B2MML schema have been translated into an approach of a vendor independent semantic model for efficient management of Human Resources and optimization of the assignment of tasks to workers according a set of parameters, including the needs of an organization. These semantically enhanced elements were used towards providing an improved balance in the workforce allocation at industrial shop floors. The concept has been successfully applied in the relevant of Systems Sunlight S.A., one of the global leaders in battery manufacturing company, which is located in Greece.

\section{$7 \quad$ Acknowledgements}

This work was partially supported by the EU funded SATISFACTORY IA project (H2020-636302). 


\section{$8 \quad$ References}

1. Berners-Lee, T., Hendler, J., \& Lassila, O. (2001). The semantic web. Scientific american, 284(5), 28-37.

2. Tsolakis, A., Arena, D., Krinidis, S., Perdikakis, A., Ioannidis, D., Kyritsis, D., \& Tzovaras, D. (2016, July). Semantically enriched industry data \& information modelling: A feasibility study on shop-floor incident recognition. In Industrial Informatics (INDIN), 2016 IEEE 14th International Conference on (pp. 487-491). Ieee.

3. Business To Manufacturing Markup Language (MESA International). http://www.mesa.org/en/b2mml.asp

4. ISA95, Enterprise-Control System Integration. https://www.isa.org/isa95/

5. Pipero, C., \& Manjunath, K. (2006, March). ISA 95 Implementation best practices: Workflow descriptions using B2MML. In WBF North American Conference, Atlanta, GA.

6. Kiritsis, D. (2013). Semantic technologies for engineering asset life cycle management. International Journal of Production Research, 51(23-24), 7345-7371.

7. Bajenaru, L., \& Smeureanu, I. (2015). An ontology based approach for modeling e-learning in healthcare human resource management. Economic computation and economic cybernetics studies and research, $49(1)$.

8. García-Barriocanal, E., Sicilia, M. A., \& Sánchez-Alonso, S. (2012). Computing with competencies: Modelling organizational capacities. Expert Systems with Applications, 39(16), 12310-12318.

9. El Kadiri, S., \& Kiritsis, D. (2015). Ontologies in the context of product lifecycle management: state of the art literature review. International Journal of Production Research, 53(18), 5657-5668.

10. Klyne, G., \& Carroll, J. J. (2004). Resource description framework (RDF): Concepts and abstract syntax. W3C Recommendation, 2004. World Wide Web Consortium, http://w3c. org/TR/rdf-concepts.

11. Maedche, A., \& Staab, S. (2001). Ontology learning for the semantic web. IEEE Intelligent systems, 16(2), 72-79.

12. Protocol, S. P. A. R. Q. L. (2016). RDF Query Language. RDF Data Access Working Group (DAWG) Std. 